\title{
Erratum to "Watery and dark axons in Wallerian degeneration of the opossum's optic nerve: different patterns of cytoskeletal breakdown?" [An Acad Bras Cienc 73(2001): 231-243]
}

\author{
MARCELO S. NARCISO ${ }^{1}$, JAN NORA HOKOÇ ${ }^{2}$ and ANA M. B. MARTINEZ ${ }^{1}$ \\ ${ }^{1}$ Departamento de Histologia e Embriologia, \\ Instituto de Ciências Biomédicas, CCS - UFRJ, 21941-590 Rio de Janeiro, RJ. \\ ${ }^{2}$ Laboratório de Neurobiologia da Retina, Instituto de Biofísica Carlos Chagas Filho, \\ Centro de Ciências da Saúde, Universidade Federal do Rio de Janeiro, Brasil
}

The Editor and the Publisher regret that Figures 1 to 4 in this article were printed with a reduction incompatible with the required resolution in electromicrographs. They apologize for any inconvenience resulting from this error. The figures are being reproduced at larger sizes and at resolutions more compatible with the originals in the following pages.

\footnotetext{
Fig. 1 - Ultrathin sections of opossum's optic nerve fibers, 24 (A and B) and 48 hours (C and D) after crush. A. Normal fibers (n) are seen among some altered fibers, which exhibit watery degeneration (star) and myelin sheath breakdown (thick arrow). Note demyelinated fibers (thin arrows) with an apparently intact axoplasmic cytoskeleton. Asterisk, astrocytic processes. Bar $=1.7 \mu \mathrm{m}$. B. Normal looking optic nerve fiber (n) is seen above an optic fiber showing dark degeneration (star). Bar $=0.4 \mu \mathrm{m}$. C. Longitudinal section of optic nerve fibers showing areas of focal degeneration of the axoplasmic cytoskeleton (star). Asterisk, astrocytic processes; m, mitochondrial profile. Bar $=0.6 \mu \mathrm{m}$. D. Observe optic nerve fibers with an apparently normal appearance (n). Among them, there is a fiber with dense axoplasm and some altered organelles, typical of dark degeneration (d). Asterisks, astrocytic processes. Bar $=0.6 \mu \mathrm{m}$.
}

Correspondence to: Dr. Ana M.B. Martinez

E-mail: martinez@ufrj.br 


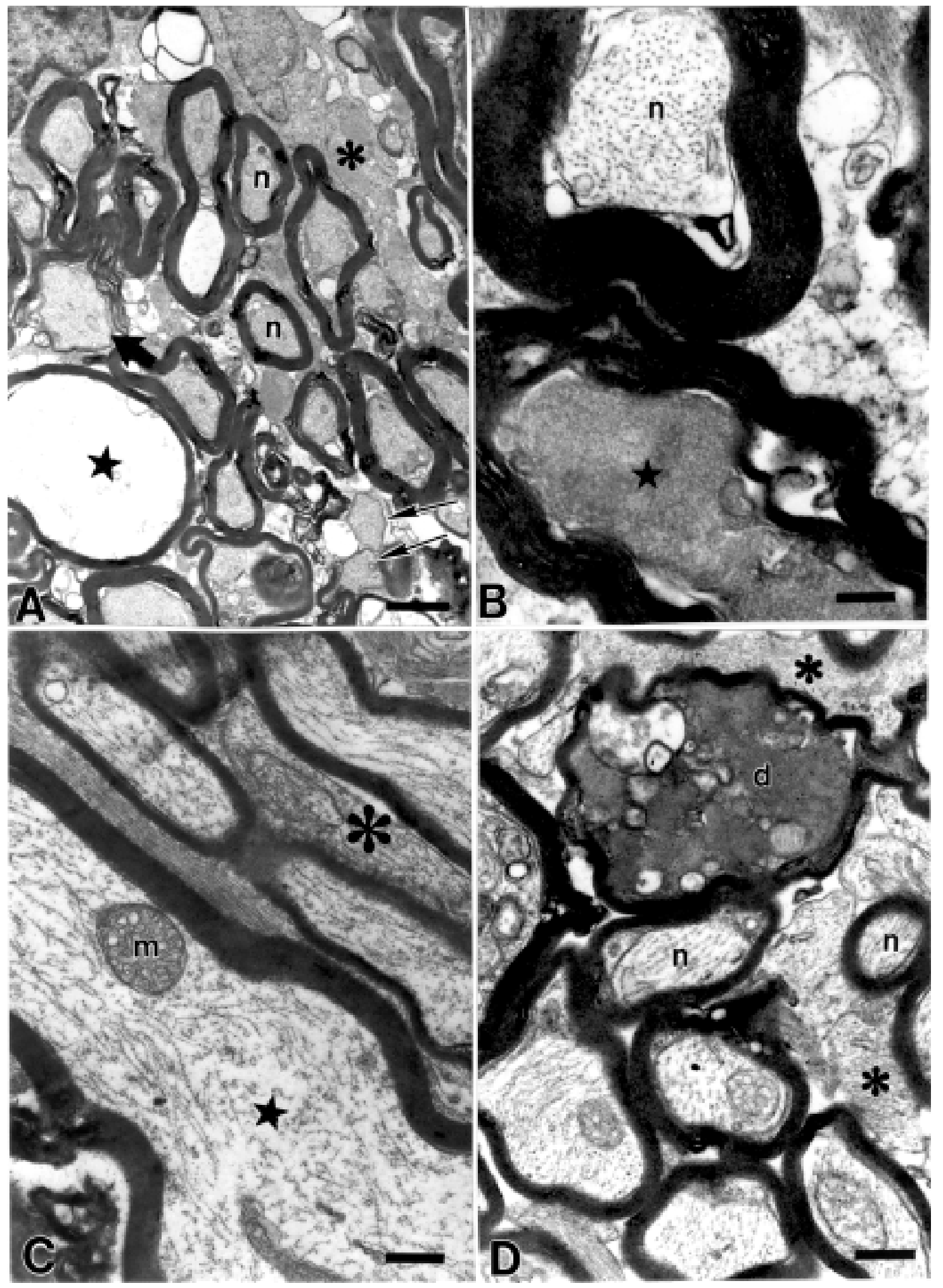




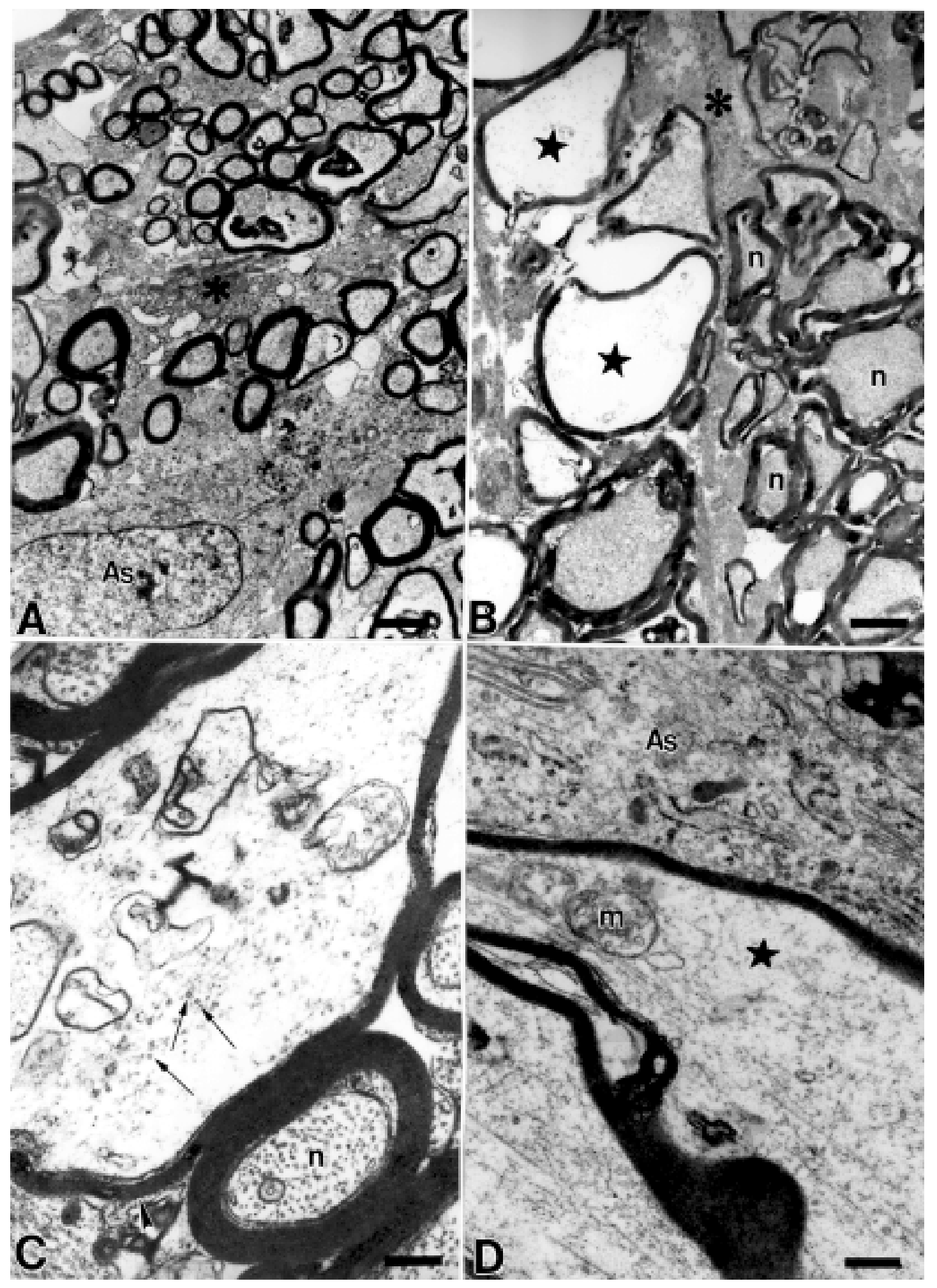


Fig. 2 - Electron micrographs of opossum's optic nerve fibers undergoing Wallerian degeneration, 72 hours after crush. A. General view of the optic nerve. Note the profuse astrocytic septa (asterisk) causing a severe disorganization of the optic fibers. Many fibers have an apparently normal appearance in this field. As, astrocyte nucleus. Bar $=1.7 \mu \mathrm{m}$. B. Optic nerve fibers exhibiting an apparently normal axoplasm (n), but there is some distortion of their myelin sheath. We can also observe a fascicle in which fibers are undergoing watery degeneration (stars) and show increased volume with no discernible organelle in the axoplasm. Asterisk, astrocytic processes. Bar $=1.7 \mu \mathrm{m}$. C. Optic nerve fiber showing reduction of axoplasmic content associated with presence of altered membranous organelles. Few microtubules (small arrows) can be seen in the axoplasm. The myelin sheath (arrowhead) is thin due to the increased axoplasmic volume, suggesting watery degeneration. Note a small-sized fiber (n) with an apparently normal appearance. Bar $=0.4 \mu \mathrm{m}$. D. Longitudinal section of optic nerve fibers showing areas of focal degeneration represented by rarefaction of the cytoskeleton (star). Some components of the cytoskeleton can still be seen in the axoplasm. An astrocytic soma (As) is also seen. $\mathrm{m}$, mitochondrial profile. Bar $=0.6 \mu \mathrm{m}$.

Fig. 3 - Electron micrographs of opossum's optic nerve fibers undergoing Wallerian degeneration 96 hours after crush. A. Thick septa with abundant astrocytic processes (asterisks) are seen among the optic fibers. Many of them exhibit an apparently normal structure (n). An oligodendrocyte (Od) is seen at the left superior quadrant of this field. Bar= $1.7 \mu \mathrm{m}$. B. A small-sized fibre is seen with the axoplasm filled with a dense and amorphous material, typical of dark degeneration (d). Normal looking fibers (n) are also observed. Bar $=0.25 \mu \mathrm{m}$. C. Periphery of the optic nerve. An optic fiber shows an extensive vacuolization and consequent delamination of myelin lamellae (arrows); the axoplasmic cytoskeleton is apparently preserved in this fiber. Normal looking fibers (n) are also observed. Portions of somata of an oligodendrocyte (Od) and a microglial cell (Mi) can be seen next to the glia limitans (gl) in this field. f, fibroblast in pia mater. $\mathrm{Bar}=1.7 \mu \mathrm{m}$. D. A large astrocytic process contains lamellar debris (thick arrows) within its cytoplasm. Observe bundles of filaments (thin arrows) in the cytoplasmic process, typical of astrocytes. Normal (n) and altered (star) fibers (with axolemmal detachment from myelin) are seen. Bar $=1.1 \mu \mathrm{m}$. 


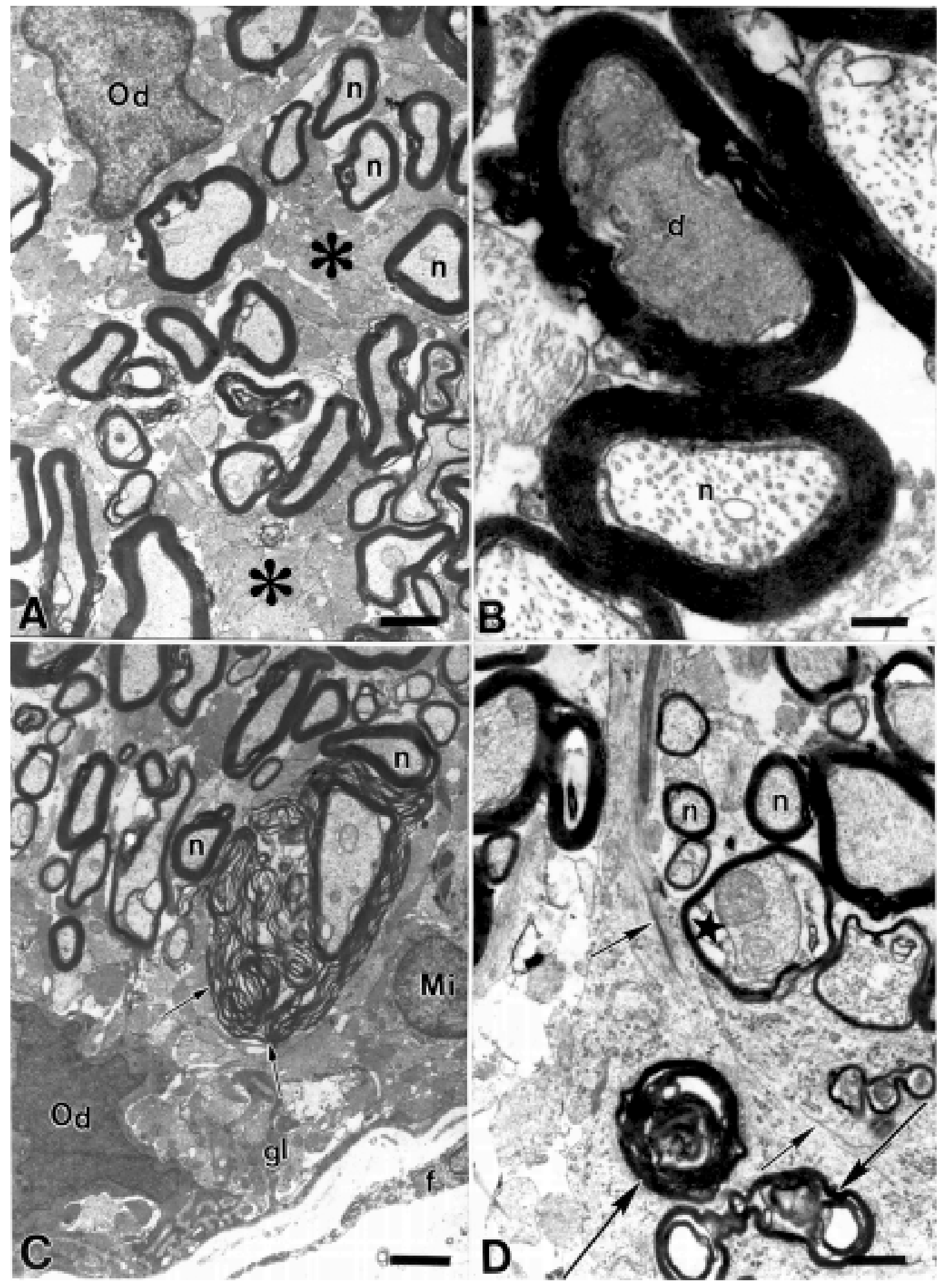




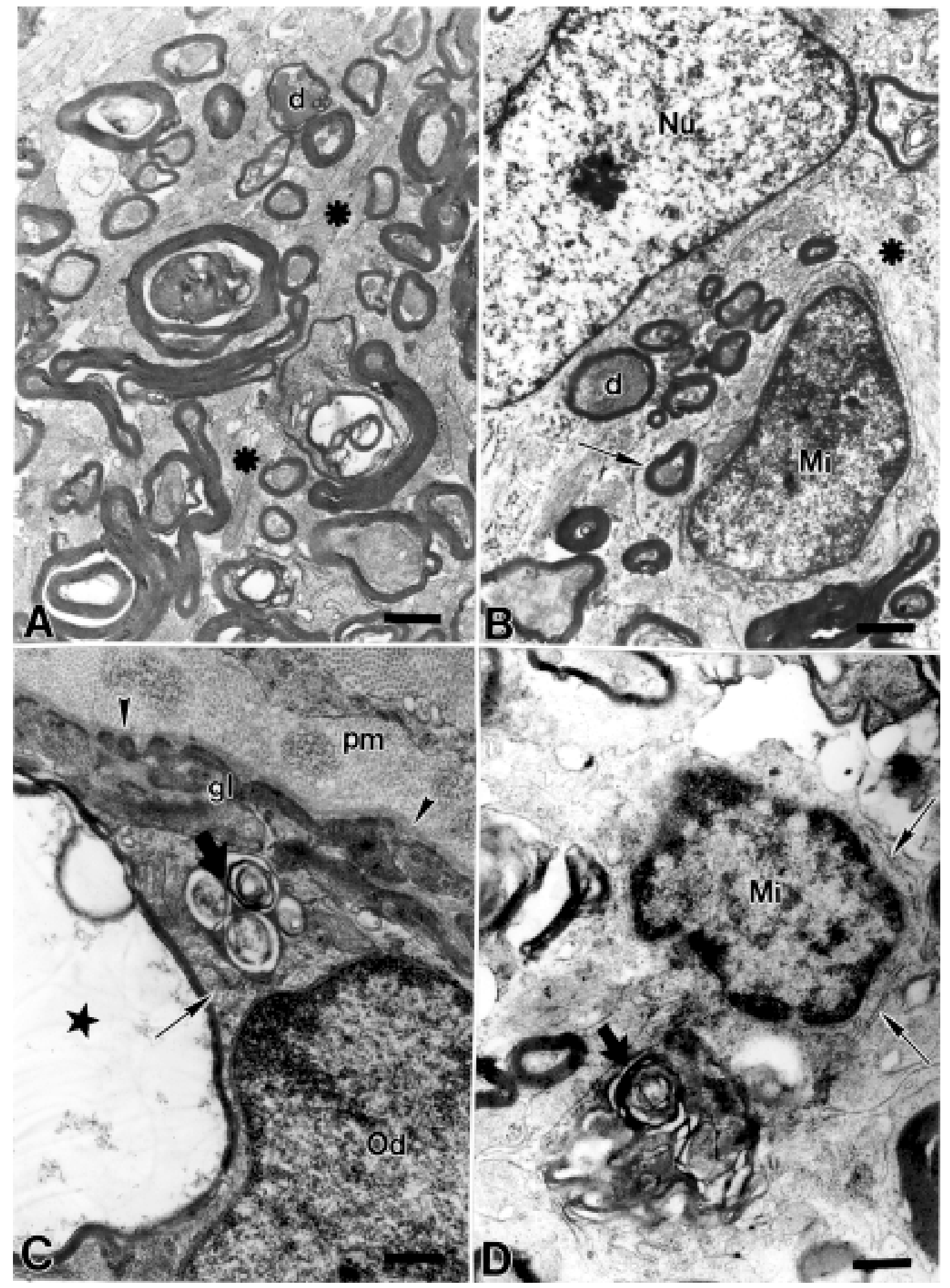


Fig. 4 - Electron micrographs of opossum's optic nerve fibers undergoing Wallerian degeneration, 168 hours after crush. A. Presence of many astrocytic processes (asterisks) causing distortion of the nerve fascicles. Note fibers with a dense axoplasm, typical of dark degeneration (d). Bar $=1.1 \mu \mathrm{m}$. B. A hypertrophic astrocyte, with thick hyperfilamentous processes (asterisk) and an euchromatic nucleus $(\mathrm{Nu})$ showing an evident nucleolus, is visualized in this field next to a microglial cell (Mi), which has scanty cytoplasm and a more heterochromatic nucleus. Note many fibers undergoing dark degeneration $(\mathrm{d})$, and one of them seems to be engulfed by the microglia (arrow). Bar $=1.1 \mu \mathrm{m}$. C. Periphery of the optic nerve, in which we can observe astrocytic processes of the glia limitans (gl) abutting the basal lamina (arrowheads). Observe an enlarged fibre (star), with a pale axoplasm, devoid of organelles, showing typical watery degeneration. Next to it, is an oligodendrocyte (Od), whose cytoplasm exhibits many microtubules (thin arrow), and presents vacuoles containing myelin debris (thick arrow). pm, pia mater. Bar $=0.6 \mu \mathrm{m}$. D. Microglial cell (Mi) with much phagocytosed myelin debris (thick arrow) within its cytoplasm. Observe the slender REG cisternae (thin arrows) around the nucleus. Degenerating fibers and myelin debris occur in the neighbourhood. Bar $=0.6 \mu \mathrm{m}$. 